\title{
Manipulating cocrystal size and morphology using a combination of temperature cycling and additives
}

Francesco Civati $^{\mathrm{a}}$, Vaclav Svoboda ${ }^{\mathrm{b}}$, Stephanie J. Urwin ${ }^{\mathrm{c}}$, Patrick McArdle ${ }^{\mathrm{a}}$, Andrea

Erxleben ${ }^{\mathrm{a},}{ }^{*}$, Denise Croker ${ }^{\mathrm{d}}$, Joop H. ter Horst ${ }^{\mathrm{c}, *}$

a Synthesis and Solid State Pharmaceutical Centre (SSPC) and School of Chemistry, National University of Ireland Galway, Galway, Ireland

b EPSRC Centre for Innovative Manufacturing in Continuous Manufacture and Crystallisation (CMAC), Department of Chemical and Process Engineering, James Weir Building, University of Strathclyde, 75 Montrose Street, Glasgow, G1 1XJ, UK

c EPSRC Centre for Innovative Manufacturing in Continuous Manufacture and Crystallisation (CMAC), Strathclyde Institute of Pharmacy and Biomedical Sciences, Technology and Innovation Centre, University of Strathclyde, 99 George Street, Glasgow, G1 1RD, UK

d Synthesis and Solid State Pharmaceutical Centre (SSPC) and Bernal Institute, University of Limerick, Castletroy, Limerick, Ireland

\begin{abstract}
Cooling crystallization of benzoic acid and isonicotinamide in ethanol yields the 1:1 cocrystal with an extreme needle-like morphology with an initial mean aspect ratio of around 10 and a size around $64 \mu \mathrm{m}$. We demonstrate that the use of suspension temperature-cycling in combination with tailor-made additives alleviates such extreme needle-like morphologies and increases the average particle size of this cocrystal material. Temperature cycling of the cocrystal suspensions in ethanol alone reduces the mean aspect ratio from 10 to 3.3 while it increases the average crystal size from 64 to $450 \mu \mathrm{m}$. The further addition of low concentrations of benzamide or nicotinamide suppresses the growth rate at the tip of the needle even more, resulting in a more favorable equant morphology. An iterative mechanism in which additives are incorporated in the lattice structure and released during the temperature increase in each cycle is proposed. Thus, the incorporation of additive at the normally fast growing and potential needle tips and its release during the temperature increase part of the cycle effectively makes additive action catalytic. The simultaneous use of temperature cycling and tailor-made additives offers a new and effective approach for the elimination of unsatisfactory needle-like crystal morphologies and a small crystal size during the production of a pharmaceutical cocrystal material.
\end{abstract}




\section{Introduction}

Crystal size and shape have a profound effect on the final product properties of pharmaceuticals. $^{1,2}$ Post-crystallization, needle-like crystal shapes pose problems during further downstream processing, as their suspensions are notoriously difficult to filter, they have bad flow properties and their ease of breakage leads to fines generation. ${ }^{3-5}$ It has been suggested that making an active pharmaceutical ingredient (API) part of a cocrystal can address many of these problems. ${ }^{6}$ In one report the application of cocrystal formation to the

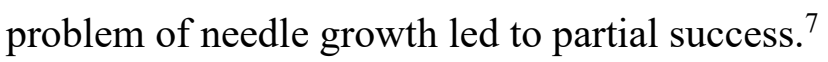

It is challenging to control both particle shape and size in a single unseeded batch cooling crystallization process, as process parameters can effect solid composition, crystal structure and particle size distribution.

A subsequent process treatment of the final suspension potentially alleviates any off-spec product. One such possible treatment is subjecting the suspension to temperature cycles after crystallization. ${ }^{8,9}$ For instance, temperature cycling suspensions of sulfathiazole has been shown to increase mean particle size through an Ostwald ripening mechanism. ${ }^{10} \mathrm{~A}$ simulation method for describing such an effect suggested that the frequently observed particle size increase or coarsening is attributed to an isothermal holding step at the maximum temperature during the temperature cycling. ${ }^{11}$ Suwannasang et al. studied a deracemization process using only temperature cycling and without the crystal grinding normally required for Videma ripening. They found that the dissolution of smaller crystals and growth of larger ones took place during temperature cycling alone. ${ }^{12}$ In another example of deracemization due to temperature cycling alone it was found that temperature cycling was more effective than standard Videma ripening with crystal grinding. ${ }^{13}$

Additives, despite been present in small quantities, can drastically impact the quality of the final crystalline product. ${ }^{14,15}$ Tailor-made additives have a close structural relationship to the target system, allowing them influence specific parts of the crystallization process. ${ }^{16,15}$ For instance, the use of in situ generated additives in the crystallization of (2-(3-(4hydroxystyryl)-5,5-dimethylcyclohex-2-enylidene)malononitrile) induced a morphological change which enhanced the suitability of the crystals for photonic applications. ${ }^{17}$ A range of additives induced a reproducible change in a diversity of crystal morphologies for methyl paraben crystals in contrast to an inconsistent and wide range of morphologies in the absence of additives. ${ }^{17,18}$ Further, it was recently found that the use of an additive in the crystallization 
of paracetamol led to a significant increase in its compressibility which improved the solid properties of the final product. ${ }^{19}$

To our knowledge, there are only a few reported examples that combine the use of temperature cycling and tailor-made additives: A polymer additive and temperature cycling of succinic acid crystals resulted in a change of the crystal shape from plate to diamond-like and temperature cycling combined with PEG and PPG polymer additives where used to modify the crystal morphology of Lovastatin..$^{20,21}$ Here, we aim to develop an approach to control product shape and size by the combined use of temperature cycling and small molecule tailor-made additives. The model compound system that is examined here is the strongly needle-like 1:1 cocrystal of benzoic acid (BZA) and isonicotinamide (INA). ${ }^{22}$ Besides the thermodynamically stable 1:1 cocrystal that crystallizes from stoichiometric solutions in ethanol, a metastable 2:1 cocrystal of BZA and INA has been reported. ${ }^{23}$

\section{EXPERIMENTAL SECTION}

\subsection{Materials and methods}

Benzoic acid (BZA), (99.5\%), benzamide (BEN) (99\%), isonicotinic acid (INIC) (99\%), isonicotinamide (INA) (99\%), nicotinamide (NA) (99\%), nicotinic acid (NIC) (99\%), 4amminobenzoic acid (PABA) (99\%), picolinamide (PIC) (99\%) and ethanol (99.8\%) were purchased from Sigma-Aldrich, Gillingham, UK and used without further purification.

\subsection{Solubility of BZA-INA in ethanol}

Using the phase diagram for the BZA-INA system ${ }^{17} 24$ a series of samples containing specific amounts of BZA-INA co-crystal in ethanol were prepared, see Figure S1. The samples were placed in a Crystalline Reactor System (Technobis, Netherlands) and heating and cooling ramps were applied. The starting temperature in this process was $13.7{ }^{\circ} \mathrm{C}$ and the maximum temperature was $55^{\circ} \mathrm{C}$ while a heating or cooling rate of $0.16^{\circ} \mathrm{C} \mathrm{min}{ }^{-1}$ and a waiting time of $30 \mathrm{~min}$ at low and high temperature to guarantee sample equilibration were applied. The clear and cloud point temperatures of the sample were determined from the recorded light transmission through the sample. Solid samples were collected through suspension filtration and analysed using XRPD Bruker D8 ADVANCE II to validate the formation of the 1:1 cocrystal (CSD code BUDWEC).

\subsection{Small Scale Cooling crystallization and temperature cycling}

To facilitate sample preparation a batch of the 1:1 co-crystal was prepared and used to prepare solutions. $^{25}$ The BZA-INA co-crystal, $0.675 \mathrm{~g}$, was dissolved in $6.525 \mathrm{~g}$ of ethanol 
(equal to $8.26 \mathrm{~mL}$ ), creating an overall composition of $81.71 \mathrm{mg} / \mathrm{mL}$ with a saturation temperature of $45^{\circ} \mathrm{C}$. The cooling crystallization process was performed using a Crystalline Reactor System (Technobis). The crystallizing solution was monitored through an integrated camera. The slurry was equilibrated for $10 \mathrm{~min}$ at $20{ }^{\circ} \mathrm{C}$ at a stirring speed of $1100 \mathrm{rpm}$ and then heated to $55{ }^{\circ} \mathrm{C}$ where it was held for $10 \mathrm{~min}$ in order to completely dissolve the solid material. The solution was then cooled to $25{ }^{\circ} \mathrm{C}$ at a rate of $0.16{ }^{\circ} \mathrm{C} / \mathrm{min}$, during which a sharp drop in light transmission was observed upon co-crystal formation.

A subsequent temperature cycling experiment was performed between 25 and $40{ }^{\circ} \mathrm{C}$ with 10 min isothermal holds at both the upper and lower temperatures. ${ }^{26}$ The heating and cooling rates used were $2{ }^{\circ} \mathrm{C} / \mathrm{min}^{-1}$ and $0.16^{\circ} \mathrm{C} \mathrm{min}^{-1}$, respectively. A slow cooling rate was selected because the decrease in temperature is directly correlated to an increase in supersaturation. Fast cooling rates lead to a high supersaturation value which would promote nucleation over crystal growth during the cooling step. Furthermore, we chose a fast heating rate to maximize the selective dissolution of fines ${ }^{5,27,28}$ To increase the impact of temperature cycling, we designed the dissolution cycle to dissolve $77 \%$ of the material. It has been reported that a high dissolution fraction can magnify the beneficial effect of temperature cycling. ${ }^{12}$ The same temperature cycling was applied to samples with different additive concentrations. The concentrations used were $0,0.7,5,10,20,30$ and $40 \%$ of the BZA-INA co-crystal used

\subsection{Larger Scale Cooling crystallization and temperature cycling experiments}

For the larger scale experiments in a $100 \mathrm{~mL}$ Easymax reactor 102 Advanced ( Mettler Toledo ) was used containing $80 \mathrm{~mL}$ of solution and a stainless-steel three-bladed overhead stirrer with an agitaion rate of $300 \mathrm{rpm}$. The BZA-INA and additive concentrations as well as the heating and cooling rates were identical to those used in the $10 \mathrm{~mL}$ scale experiments. The monitoring using online FBRM (Mettler Toledo) allowed measurement of crystal chord length distribution and particle count. The FBRM (Mettler Toledo) used a monochromatic laser beam with a rotation speed of $2 \mathrm{~m} \mathrm{~s}^{-1}$. The focal point was constant for all the measurements and time and data points were collected every 2 seconds. ${ }^{22,29}$

\subsection{Cocrystal Product Analysis}

Samples were analyzed by XRPD using either a Bruker D8 ADVANCE II using a $2 \theta$ range of 5 to $40{ }^{\circ}(\mathrm{Cu} \mathrm{K \alpha} 50 \mathrm{kV} 50 \mathrm{~mA})$ in transmission mode or an Inel Equinox 3000 with a $2 \theta$ range of 4 to $80^{\circ}(\mathrm{Cu} \mathrm{K \alpha} 35 \mathrm{kV} 25 \mathrm{~A})$ in reflection mode.

NMR was used to quantify the amount of BEN and PABA additives incorporated into the final cocrystal product. The final suspensions were filtered and washed with ethanol and 
dried in air prior to their dissolution in DMSO- $\mathrm{d}_{6}$ for analysis. ${ }^{1} \mathrm{H}-\mathrm{NMR}$ spectra were recorded on a Varian $500 \mathrm{MHz}$ instrument and chemical shifts were reported relative to the residual DMSO- $\mathrm{d}_{6}$ solvent signal $(2.50 \mathrm{ppm})$. In the case of BZA-INA samples with the additive PABA, the selected signals for analysis were a PABA multiplet at $6.54-6.50 \mathrm{ppm}$ and an INA multiplet at $8.71-8.69 \mathrm{ppm}$. For the BZA-INA samples with the additive BEN the selected signals were a BEN multiplet at $7.87-7.84 \mathrm{ppm}$ and an INA multiplet at $8.71-$ $8.69 \mathrm{ppm}$. It was not possible to determine the amount of NA using this method due to resonance overlap.

A Renishaw Invia micro-Raman spectrometer was used to obtain Raman spectra in the 100 $-3600 \mathrm{~cm}^{-1}$ range, with $4 \mathrm{~cm}^{-1}$ resolution, $10 \mathrm{~s}$ exposure time and $0.5 \%$ laser power using a $785 \mathrm{~nm}$ laser. FT-IR spectra were collected on a Perkin Elmer Spectrum 400 fitted with an ATR reflectance attachment. IR spectra were collected in the range of $650-3600 \mathrm{~cm}^{-1}$, with a resolution of $4 \mathrm{~cm}^{-1}$ and four scans on a diamond/ZnSe window.

Throughout this paper aspect ratio is defined as maximum length over minimum width of the $2 \mathrm{D}$ projection in a photographic image. A minimum of 50 crystals from the in-situ camera images were used to calculate mean values for crystal aspect ratio and crystal length.

\subsection{Co-crystal Structure Analysis}

The Pixel program (June 2018 version http://www.angelogavezzotti.it/.) ${ }^{23} 30$ was used to calculate intermolecular energies in the lattice. Pixel operations and the operation of Gaussian16w for electron density calculations were automated by Oscail ${ }^{24}$ using CSD code BUDWEC with the C-H bond lengths set to 1.08 and N-H and $\mathrm{O}-\mathrm{H}$ bond lengths set to $1.0 \AA$. Centroid distance matrix analysis of the BZA-INA structure was carried out using the Oscail software package. ${ }^{31}$

\section{Results}

As previously reported, cooling a 1:1 stochiometric mixture of BZA and INA in ethanol resulted in the crystallization of a BZA-INA co-crystal product. ${ }^{25}$ The formation of a single crystalline phase corresponding to the BZA-INA 1:1 co-crystal was confirmed by XRPD analysis (Figures S3). ${ }^{22,32,33}$ The solubility and metastable zone of the resulting co-crystal in ethanol were determined were determined using multiple samples with a range of concentrations using turbidity measurements, and are shown in figure 1(b). A strongly elongated morphology of the co-crystal product was evident, figure 1(a). Based on the preliminary cocrystallization experiments, a co-crystal concentration of $81.7 \mathrm{mg} / \mathrm{mL}$ in 
ethanol having a saturation temperature of $45^{\circ} \mathrm{C}$ was selected to be convenient for the subsequent experiments. Investigation of the cooling rates indicated that rapid cooling $\left(2{ }^{\circ} \mathrm{C}\right.$ $\left.\min ^{-1}\right)$ resulted in an agglomerated product, whereas slow cooling $\left(0.16^{\circ} \mathrm{C} \min ^{-1}\right)$ gave welldefined single cocrystals.

(a)

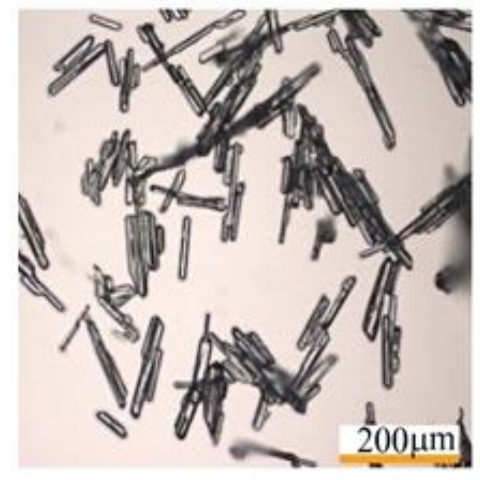

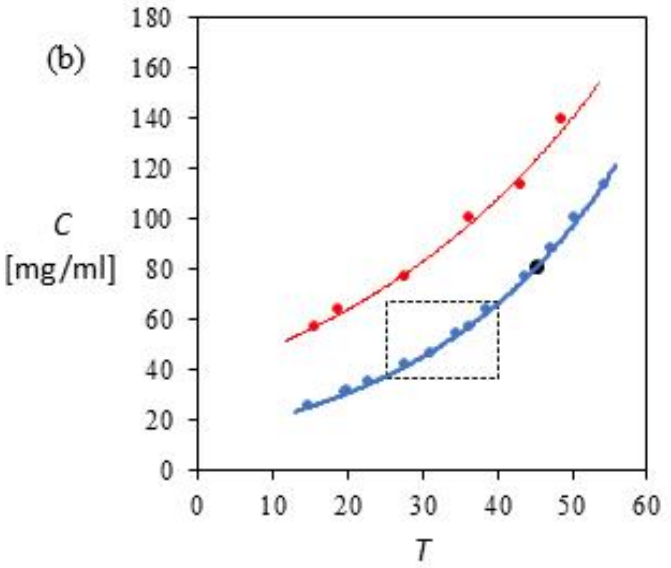

$\left[{ }^{\circ} \mathrm{C}\right]$

Figure 1. (a) BZA-INA co-crystals grown from an ethanol solution with an initial concentration of $81.71 \mathrm{mg} / \mathrm{mL}$ of co-crystal material using a cooling rate of $0.16{ }^{\circ} \mathrm{C} / \mathrm{min}$. The aspect ratio of the crystals is around 10 and the average size is $90 \square \mathrm{m}$. (b) The solubility (blue) and nucleation (red) and metastable zone region of BZA-INA in stoichiometric solutions, calculated using a polythermal method. The temperature cycle used is highlighted with black dashed lines, the temperature cycle begins at $40{ }^{\circ} \mathrm{C}$ and ends at $25^{\circ} \mathrm{C}$. The initial concentration is indicated with a black dot on the blue line at $45^{\circ} \mathrm{C}$.

\subsection{Temperature Cycling}

The general temperature profile used for the small-scale cocrystallisation of BZA-INA in the absence of additives is shown in figure $2 \mathrm{a}$. The primary nucleation event occured at $33.5^{\circ} \mathrm{C}$, close to the end of the initial cooling ramp. Immediately after nucleation, a relatively high number of small crystals were observed (Figure 2b, Image 0). Images taken during temperature cycles 2, 4, 6 and 8 show a progressive reduction in fines and an increase in crystal size with increasing cycle number. The rounded corners on crystals on images taken at the maximum cycle temperature (red squares) indicate dissolution has occurred, while those taken at the low temperature of the cycles show crystals with faceted crystals following crystal growth during cooling. After 8 successive cycles large needle crystals are observed (figure $2 b$, image 8 ). 


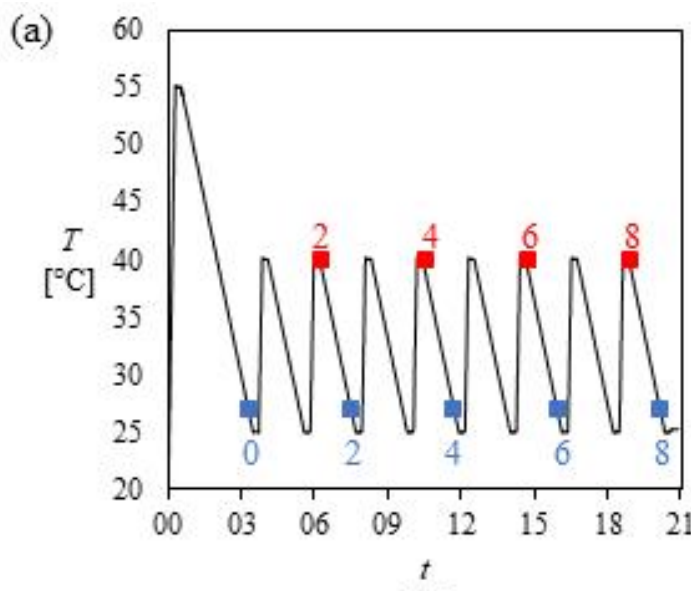

(b)

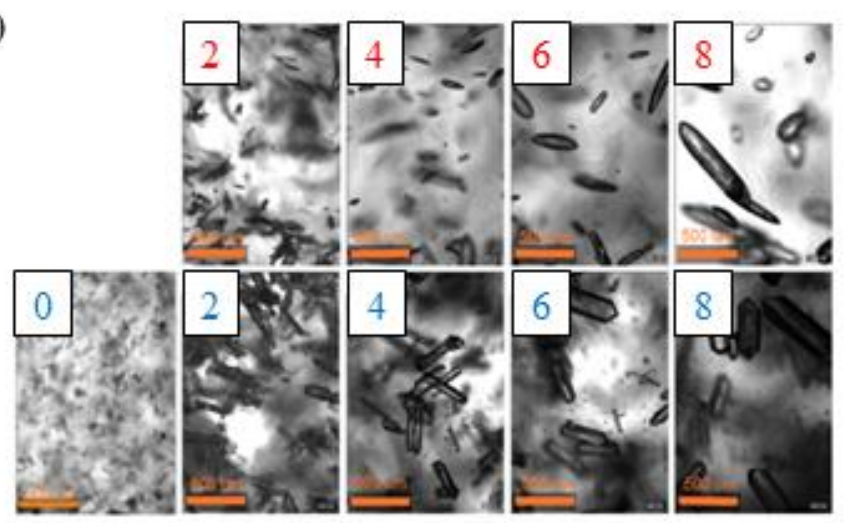

[h]

Figure 2. (a) Temperature profile used in a small scale cocrystallization / temperature cycling experiment in the absence of additives. (b) Suspension pictures taken at the points indicated by the blue and red squares in the left graph. The experiments were performed using a concentration of 81.71 $\mathrm{mg} / \mathrm{mL}$, which has a clear point temperature at $45^{\circ} \mathrm{C}$.

The conditions used for the small-scale experiments were increased 10-fold, and BZA-INA was cocrystallised on a $100 \mathrm{~mL}$ scale with temperature cycling in the absence of additives. In a typical experiment, three chord length ranges, small $(<50 \mu \mathrm{m})$, medium $(50-100 \mu \mathrm{m})$ and large $(100-1000 \mu \mathrm{m})$, were measured over time using an FBRM probe. A sudden increase in total counts, mostly of small particles, detected at $29.4{ }^{\circ} \mathrm{C}$ indicated nucleation had occurred. Once the minium temperature has been reached, the particle count in the small chord length range decreases only slightly, indicating that no significant primary or secondary nucleation events take place after the initial nucleation. During the first subsequent dissolution stage, there is a significant reduction in counts of the small, and to some extent also of the middle, chord length range, indicating the smallest particles dissolved as the suspension temperature increased. Each subsequent temperature cycle shows a similar but reducing effect. In the 8th temperature cycles only minor changes to the FBRM counts were observed. The chord length distributions in each temperature cycle collected at $28{ }^{\circ} \mathrm{C}$ during the cooling ramp are overlaid in Figure 3(b). This highlights how the total number of particles decreases and the mean chord length increases after each successive temperature cycle. Microscopy images of the needle-like crystals isolated from the 100 and $10 \mathrm{~mL}$ experiments after 8 temperature cycles are shown in Figures 3(c) and (d) for comparison. No fines were present in either sample. There was only a small difference in the aspect ratios and crystal lengths. The mean aspect ratios are $6.2 \pm 1.7$ and $3.4 \pm 1.4$ and crystal lengths are $345 \pm 77$ and $450 \pm 92$ 
respectively. In the $10 \mathrm{~mL}$ experiment temperature cycling seems to reduces the aspect ratio by a factor of 3 and increases the crystal length by a factor of 7 (Figure 3(e) and (f)). Temperature cycling has successfully increased the particle size of the BZA-INA cocrystal, but the isolated crystals still had an unwanted extreme needle-like morphology. Therefore, addition of tailor-made additives to the cocrystallisation was explored as a different method to bring the aspect ratio closer to 1 .

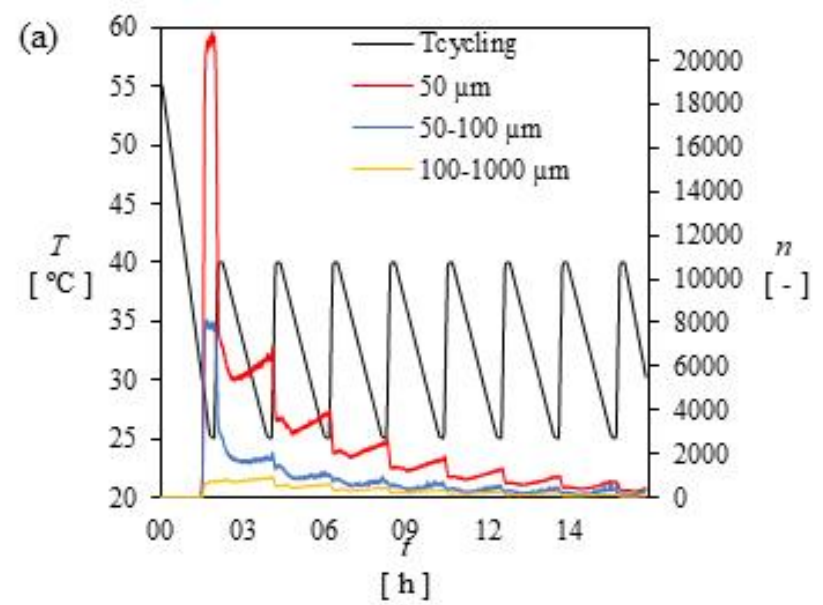

(b)

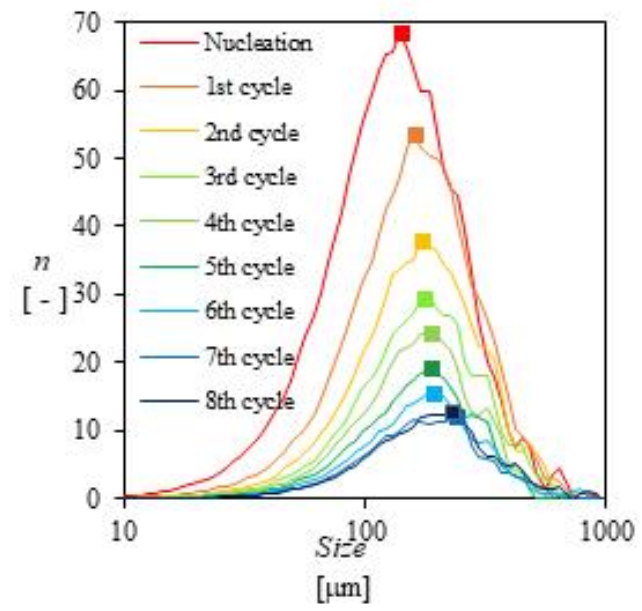

(c)

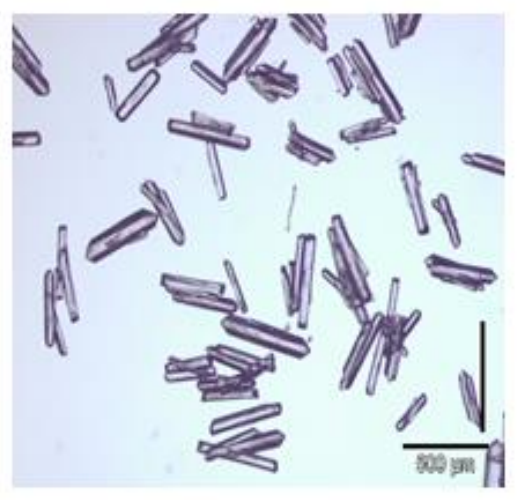

(d)

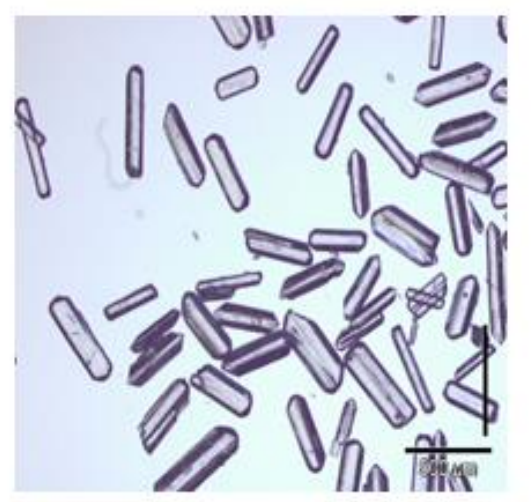

(e)

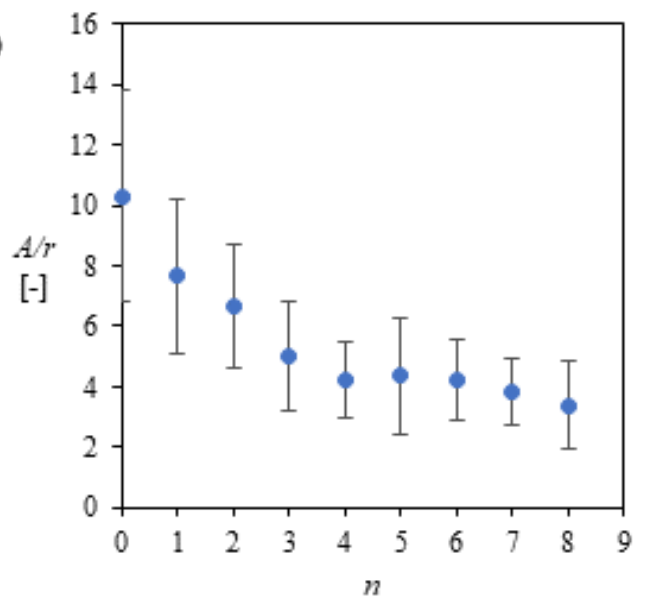

$[-]$

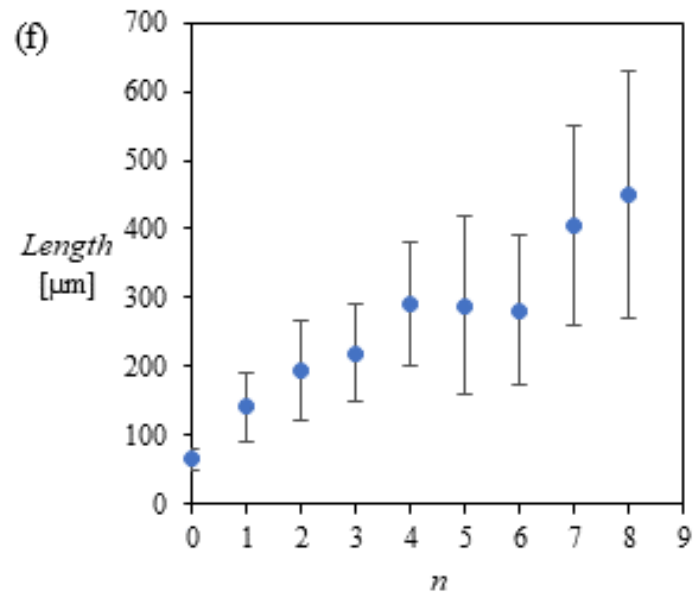

$[-]$ 
Figure 3. a) Counts in the small $(<50 \mu \mathrm{m}$, red), medium $(50-100 \mu \mathrm{m}$, blue) and large $(100-1000 \mu \mathrm{m}$, black) chord size ranges during temperature cycling of a BZA-INA co-crystal suspension in ethanol, obtained using a $100 \mathrm{~mL}$ scale EasyMax reactor. (b) FBRM counts for particle size ranges measured at $28^{\circ} \mathrm{C}$ in the cooling part of the temperature cycles in the $100 \mathrm{~mL}$ EasyMax reactor, (c) final crystal product from $100 \mathrm{~mL}$ scale and, (d) final crystal product from $10 \mathrm{~mL}$ scale, (e) aspect ratio of the cocrystal in each cycle at $30{ }^{\circ} \mathrm{C}$ and (f) crystal length variation during temperature cycling at $30^{\circ} \mathrm{C}$, using a $10 \mathrm{~mL}$ scale Crystalline Technobis apparatus.

\subsection{The Combination of Additives and Temperature Cycling}

The selection of various tailor-made additives for the isolation of the BZA-INA co-crystal is depicted in Scheme 1. The strategy used was to induce faults in the crystal structure as described in section 4.2 below. Additives were chosen which maintained the amide and carboxylic acid functionality of INA and BZA but had modifications around the phenyl rings. Initally, the six additives were screened for an effect on particle shape at a $0.7 \mathrm{w} \%$ loading in cooling crystallizations with temperature cycling, and compared with the small-scale experiments in the absence of additives (Figure 4).

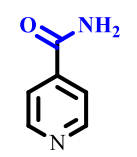

INA

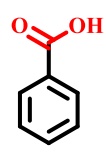

BZA

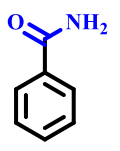

BEN

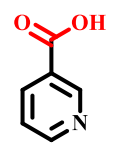

NIC

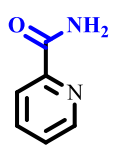

PIC

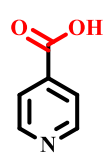

INIC
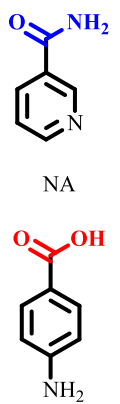

PABA

Scheme 1: Molecular structure of BZA, INA and additives BEN, PIC and NA having an amide functionality and additives NIC, INIC and PABA having an carboxylic acid functionality.

In the procedure used $0.7 \% \mathrm{w} / \mathrm{w}$ of additive was loaded into the sample before complete dissolution and then temperature cycling was started as described above. All of the additives used were examined in separate temperature cycling runs. While PIC, NIC and PABA appear to have no effect on BZA-INA co-crystal growth, visual inspection of in situ images indicated the addition of amide-modified additives BEN and NA ( $c$ and e) significantly reduced the crystal aspect ratio. Curiously, additive INIC seemingly reduces crystal growth 
significantly, Figure 4(g). Examination of the crystallisation product in all cases using XRPD, indicated only the BZA-INA cocrystal was present, Figure S3.

These thermal cycling experiments were repeated with higher initial additive concentrations to increase additive levels in the crystal product to levels which would allow analysis by Raman and FT-IR spectroscopy. More details in section 3.3.2 below. XRPD patterns of crystal products obtained with additives at $10 \%$ concentration showed that the cocrystal was the only product except in the case of INIC in which case formation of the cocrystal was completely blocked and only peaks due to INIC and a small amount of BZA were observed in the XRPD, Figure $\mathbf{S 4}$.

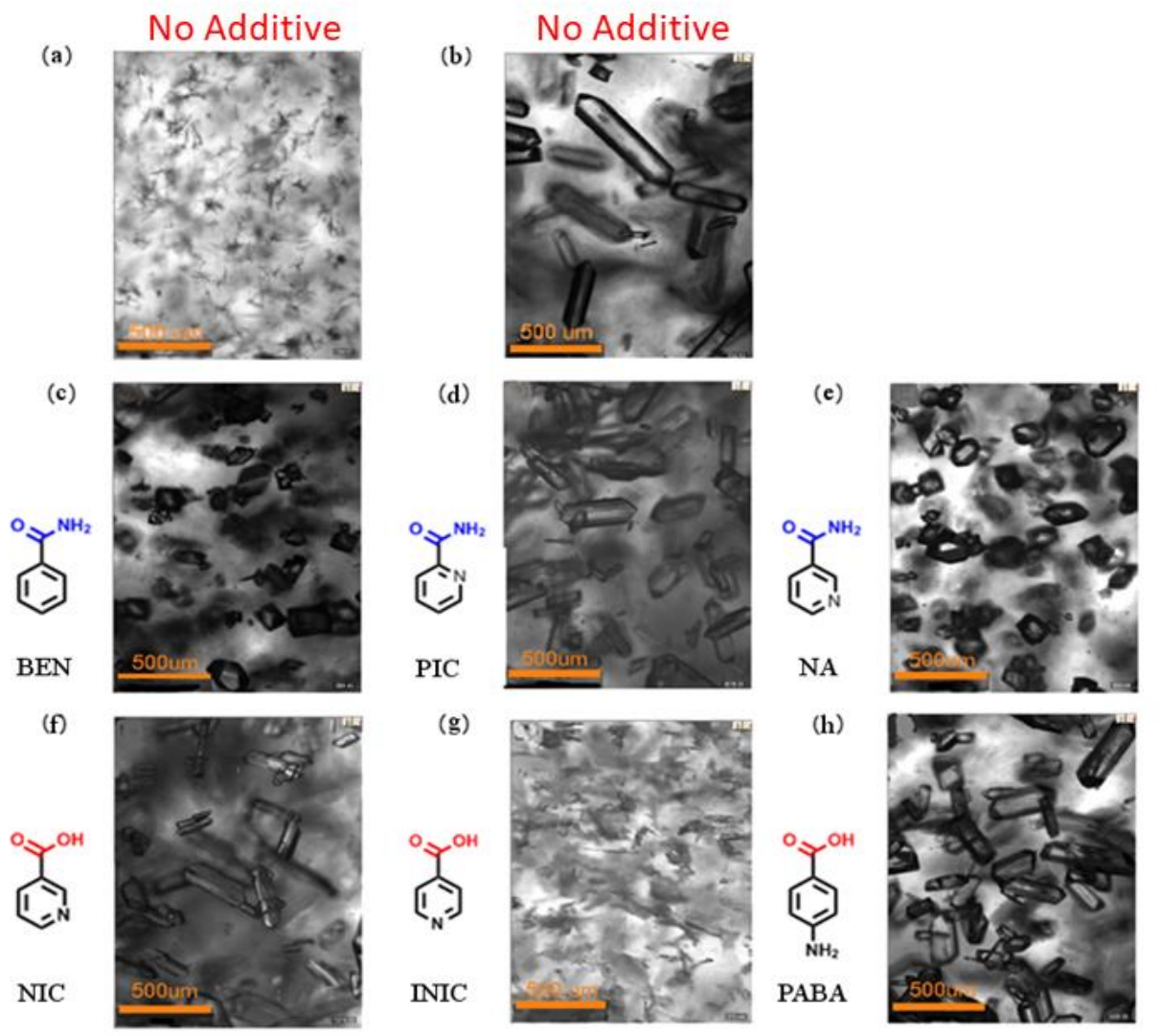

Figure 4. Small scale temperature cycling in the absence and presence of additives. In situ images of the suspension after (a) the first nucleation event without additives, (b) after 8 temperature cycles without additives, (c)-(h) after 8 temperature cycles in the presence of $0.7 \%$ of (c) BEN (d) PIC, (e) NA, (f) NIC, (g) INIC and (h) PABA. 


\subsubsection{Crystal Aspect Ratio Change}

Aspect ratios were determined using images collected in situ during crystallisation experiments. When $0.7 \mathrm{w} \% \mathrm{BEN}$ was added to the crystallization, the aspect ratio decreased from 9.90 to 1.51 over the eight temperature cycles (Figure 5a), demonstrating that near perfect control of crystal aspect ratio can be obtained when this additive is used in conjunction with temperature cycling. Increasing the additive concentration to $10 \mathrm{w} \%$ BEN suppressed nucleation and crystal growth, resulting in significantly smaller and fewer crystals. In contrast, the aspect ratios observed for runs with no additive and NIC at $0.7 \%$ are very similar, Figure $\mathbf{S 5}$.

Interestingly, the crystal aspect ratios had an observable effect on XRPD peak intensities when measured in reflection mode, likely due to preferred orientation. The intensity of the peak close to $17^{\circ}$ is enhanced in BZA-INA samples which have needle crystals, Figure S4. This peak is due to reflection from the (-4 02 2) plane, which is parallel to the needle axis. The observed intensity of the (4 0 2) peaks is closer to the calculated value in samples crystallized in the presence of BEN and NA in which needle growth was suppressed.

(a)

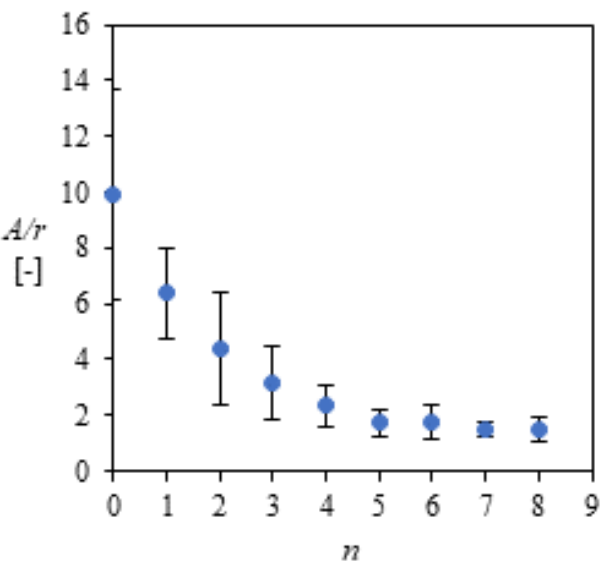

$[-]$

(c)

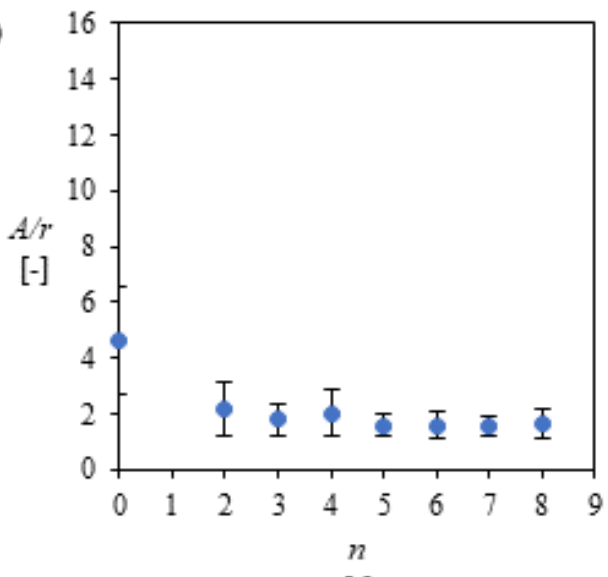

[-]

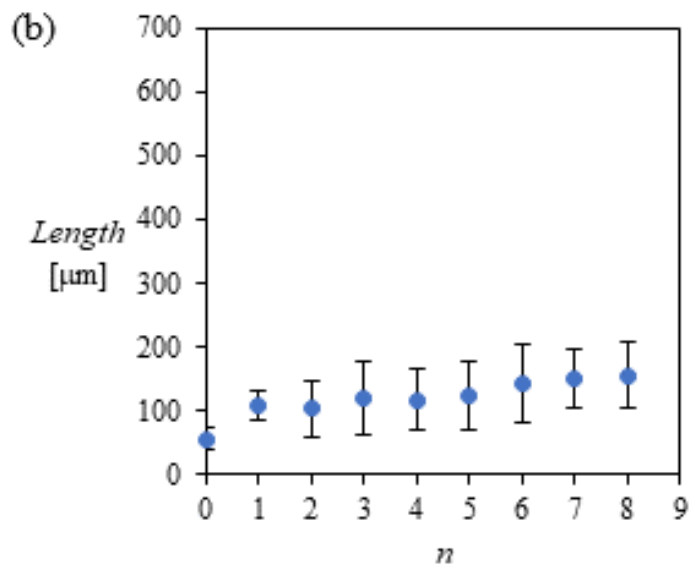

$[-]$

(d)

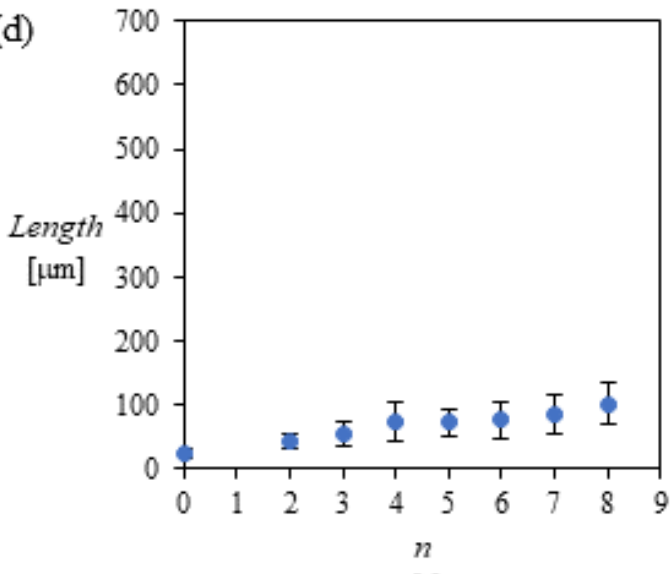

[-] 
Figure 5. Crystal aspect ratios and crystal lengths after each thermal cycle using the small scale temperature cycling set up with BEN at $0.7 \%$ a) aspect ratio and b) crystal length and with BEN at $10 \% \mathrm{c}$ ) aspect ratio and d) crystal length.

\subsubsection{Other Product Quality Aspects}

To establish if the additives were incorporated into the BZA-INA crystal structure or had crystallized concomitantly, crystals with additive concentration high enough for concentration measurement by Raman, FT-IR, XRPD and ${ }^{1} \mathrm{H}$ NMR were sought. The limit of detection for orthorhombic paracetamol in a mixture with the monoclinic form by Raman and FT-IR has been reported to be $0.012 \%$ by weight. ${ }^{33}$ For ranitidine. $\mathrm{HCl}$, the limit for detection for form II in form I is reported to be $2.2 \%$ by FT-IR and $3.4 \%$ by XRPD. ${ }^{34}$ While these detection limits are close to the lowest solution concentration of additives used of $0.7 \mathrm{w} \%$ the actual incorporated level of additives in the crystal product is likely to be lower than $0.7 \mathrm{w} \%$ as $100 \%$ incorporation of additive from solution is unlikely. For this reason, a series of experiments were carried out using higher additive levels of 5, 10, 20, 30 and $40 \mathrm{w} \%$. It was found that an additive level of $10 \%$ was sufficient to permit analysis by FT-IR and Raman spectroscopies.

Using ${ }^{1} \mathrm{H}$ NMR spectroscopy it was possible to estimate the quantity of BEN and PABA in the crystallised samples after 8 cycles, (Figures S6-8, summarized in Table 1). The distribution of additive concentration between solution and the isolated solid is non-linear and reaches a limiting value for BEN more rapidly than for PABA indicating possible concomitant crystallization in the PABA case. Additive concentrations above $10 \%$ were used only to examine the limiting nature of additive incorporation with increasing solution concentration. It is however interesting that even at $40 \%$ additive concentration the $1: 1 \mathrm{BZA}-$ INA cocrystal was the least soluble phase in the solution and as examined by PXRD the only one which crystallized.

Table 1. Additive concentration in BZA-INA determined by ${ }^{1} \mathrm{H}$ NMR spectroscopy

Additive concentration added in solution Additive detected in product by weight $\%$ of BZA-INA weight $\%$ of BZA-INA

BEN
$0.7 \%$
$5 \%$
$10 \%$
$0.14 \%$
$0.49 \%$
$0.99 \%$
$0.14 \%$
$1.39 \%$
$2.14 \%$

PABA 
The concentrations of $\mathrm{BEN}$ and $\mathrm{PABA}$ in the crystal product at $10 \%$ initial additive concentration, in the $0.5-1.4 \%$ range were high enough to be detectable by both FT-IR and Raman spectroscopies and this additive concentration of $10 \%$ was used to obtain samples for spectroscopic studies.

When the crystal products obtained with the additives BEN and NA at $10 \%$ solution concentration were examined using Raman spectroscopy, new additional absorption bands not in the spectra of BZA-INA, BEN or NA were found at 140, 690 and $1250 \mathrm{~cm}^{-1}$, Figure 6(a).

(a)

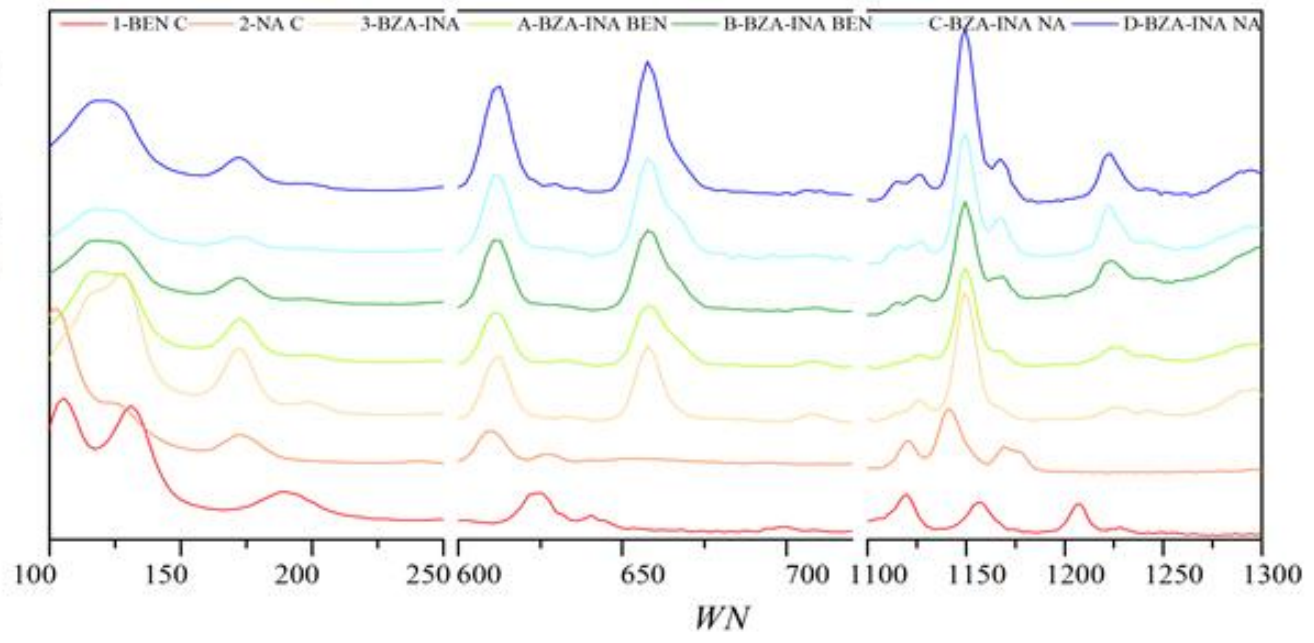

A.U.

$[-]$ $\left[\mathrm{cm}^{-1}\right]$

(b)

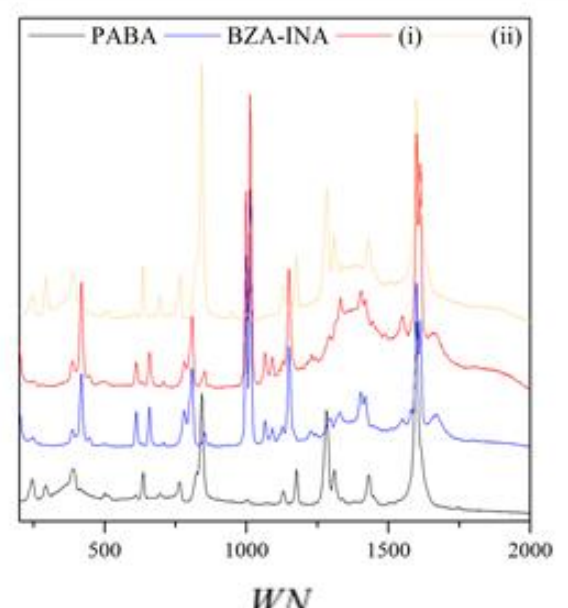

$\left[\mathrm{cm}^{-1}\right.$ ] (c)

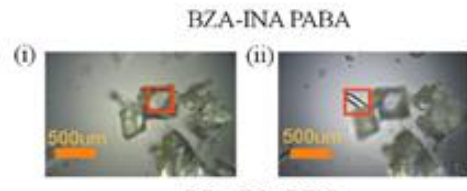

BZA-INA BEN

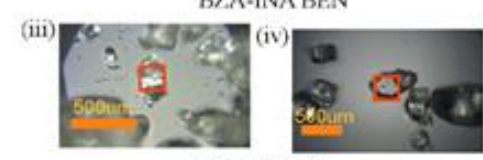

BZA-INANA

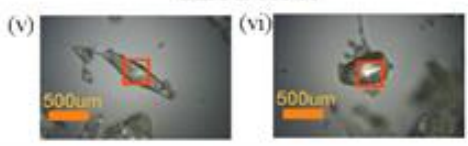


Figure 6. Raman spectra of BZA-INA cocrystals grown using a small-scale temperature cycling setup in the presence of (a) BEN and NA and (b) PABA and (c) pictures of the crystals used with the target crystal marked by a red square.

These new absorbance bands indicate additive incorporation into the BZA-INA lattice The FT-IR spectra of crystals grown in the presence of NA show a shoulder at $3427 \mathrm{~cm}^{-1}$ and smaller features at 1740 and $1720 \mathrm{~cm}^{-1}$ which are not observed in the spectra of BZA-INA or NA. This provides further support for incorporation of the additive into the BZA-INA lattice, Figure 7(a).

In contrast, where PABA was added at the same solution concentration, the resulting product sample was found to contain two types of crystals; larger crystals and smaller elongated crystals. When compared to the Raman spectra of commercial PABA and BZA-INA samples (Figure 6(b)), it was found that the larger crystal (i) and the elongated crystal (ii) correspond to the spectra of pure PABA and BZA-INA respectively. FT-IR spectra of the crystal product grown in the presence of PABA shows an additional peak at $3470 \mathrm{~cm}^{-1}$, which is in the same position as a peak in the PABA spectrum, Figure 7(b). Therefore, it was concluded that PABA was not incorporated into the BZA-INA cocrystal lattice, instead this additive crystallized concomitantly. FT-IR spectra of crystal products obtained in the presence of other additives are in Figure S9.
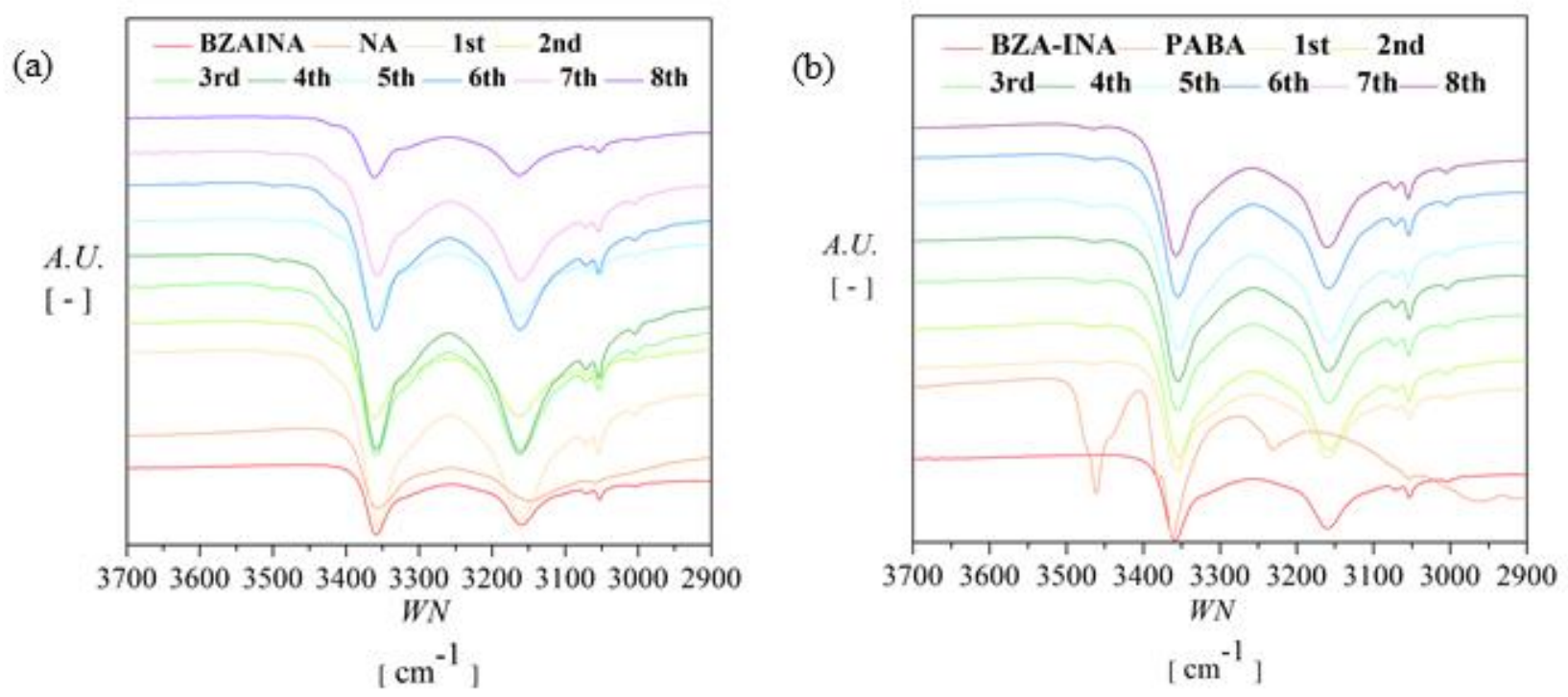

Figure 7. FT-IR spectra of BZA-INA cocrystals grown in the presence of (a) NA and (b) PABA. 


\section{Discussion}

\subsection{The origin of BZA-INA needle growth}

A typical needle shaped crystal of BZA-INA crystallised in this work with extended growth along $b$ is shown in Figure 9(a). This deviates substantially from the rather block-like calculated Bravais-Friedel-Donnay-Harker (BFDH) morphology, Figure 9(b) ${ }^{28,35}$ When the crystal shape was calculated using slice attachment energies, it has a dominant (2 0-2) face but it is also block like, Figure S2.

(a)

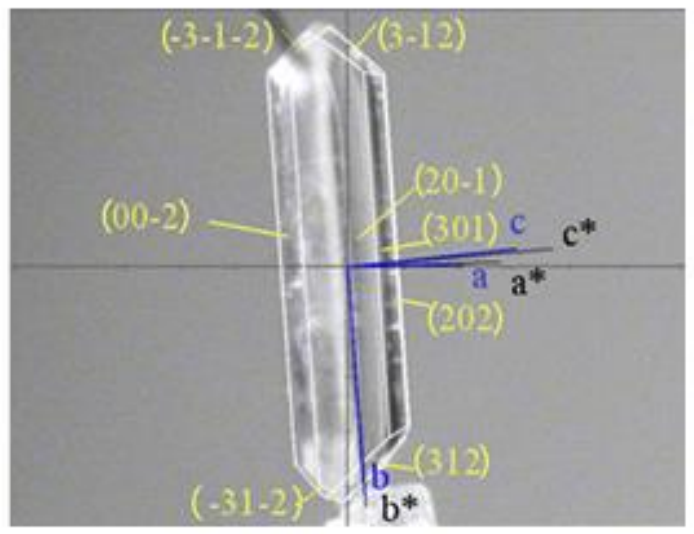

(b)

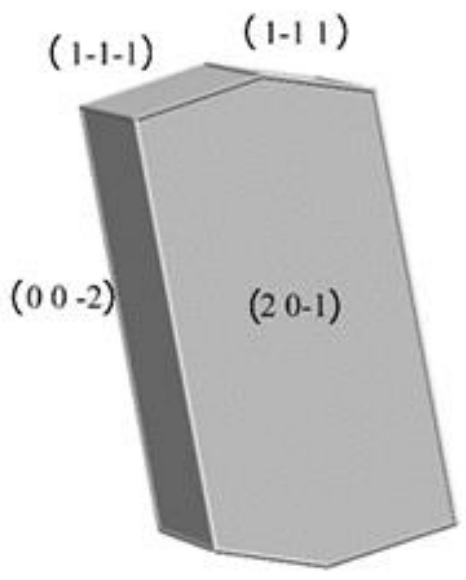

Figure 9. (a) A face-indexed BZA-INA crystal. (b) BFDH calculated BZA-INA crystal shape.

Studies of needle-shaped crystals show that growth in the elongated direction proceeds by a different mechanism to that of the larger side faces. ${ }^{36,37}$ The needle tips of $\beta$-pthalocyanine grown by sublimation and urea grown from aqueous solution show rough growth whereas the needle side faces show smooth growth. ${ }^{37,38}$ Since crystal growth and dissolution are expected to have analogous mechanisms $\mathrm{s}^{39,40}$ an insight into crystal needle growth can be obtained by examining needle dissolution. Dissolution movies of needles of carbamazepine form I and diflunisal form II show that the needles get shorter faster than they get thinner, as would be expected for the reverse of needle growth, and also that the needle tips become rough and pointed as they dissolve. ${ }^{28,41}$ Thus needle growth and dissolution in the needle direction has a kinetic advantage over growth in other directions which allows the crystals to have aspect ratios much larger than suggested by BFDH and slice attachment energies. It has been suggested that the kinetic advantage arises, in the case of stacked structures, from the ease with which molecules which are flat or nearly flat can add to the ends of stacks rather than the stack sides. ${ }^{42} \beta$-Pthalocyanine, carbamazepine form I and diflunisal form II all have 
stacked structures and high aspect ratio needle crystals. A simple measure of stacking is to count the $\%$ of atoms in a molecule that are in $\mathrm{vdW}$ contact with its neighbours above and below it in the stack. The lower the fraction of atoms in contact the greater the slippage in the stack. In a range of examples it has been observed that when this fraction is greater than $50 \%$ needle growth is observed. ${ }^{42}$

Application of this analysis to the BZA-INA structure shows that $51 \%$ of the atoms of both of the molecules in the asymmetric unit are in contact with their stack neighbours along the $b$ axis. The molecular stacking in the crystal structure of the BZA-INA co-crystal ${ }^{25}$ is shown viewed along the $b$ axis (needle growth direction) in Figure 10(a). The structure is built up from super molecules in which $R_{2}^{2}(8)$ bridged INA dimers are capped on both sides by a BZA molecule which hydrogen bonds to the pyridyl-nitrogen of the INA dimer. This linear group of 4 molecules (numbers 2-5 in Figure 10 (b) form further weaker hydrogen bonds through the amide of the INA molecule and the carbonyl oxygen of the BZA molecule to other, almost perpendicularly positioned supermolecules. This structure is broadly similar to that of benzoic acid (CSD code BENZAC11), which has H-bonded dimers in slipped stacks and also exhibits needle growth in the stacking direction.

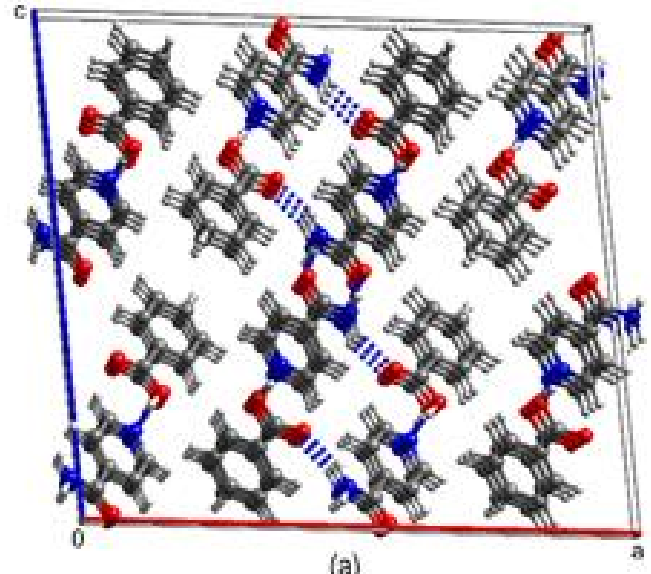

(a)

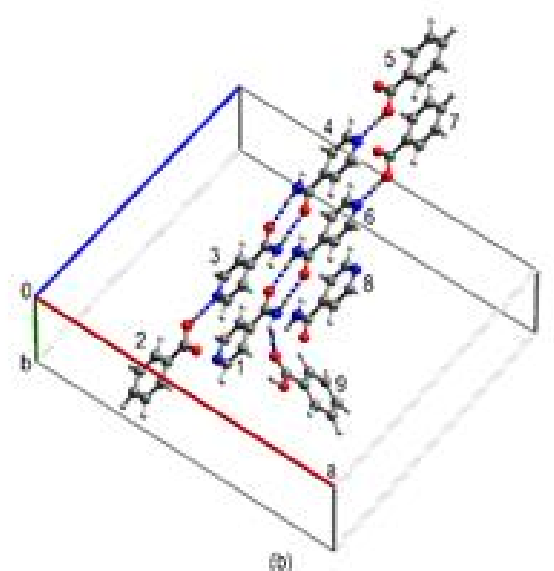

(9)

Figure 10. (a) View of the stacking in the in the BZA-INA crystal structure down the $b$ axis and (b) Hydrogen bonding and vdW interactions. The molecule numbers are used in Table 2.

The intermolecular interactions in the lattice were analyzed using Pixel. ${ }^{43}$ The intermolecular energies calculated for the seven strongest interactions and the total energies are given in Table 2. It is assumed coincidental that the numerical values for the total Coulombic and total interaction (lattice) energies are identical. Molecules referred to in Table 2 can be 
identified by their mass weighted centroid distances, their symmetry operations and the associated molecule numbers in Figure 10(b). The two strong hydrogen bonds which form the supermolecule can be easily identified, due to their dominant Coulombic content, in the interactions between molecules numbered 3 and 4 and 2 and 3 respectively in Table 2 and Figure 10(b). The weaker hydrogen bond between molecules 1 and 9 which links the supermolecules together also has a dominant Coulombic content. The other four interactions have dominant dispersion energy contributions and it is these interactions together with the 1 , 9 H-bond which bind the stack layers together. The additives selected in 3.2 above have the potential to be incorporated into the lattice and to introduce faults in the stacks which could hinder crystal growth.

Table 2 Intermolecular energies (kJmol-1) for BZA-INA structure calculated using Pixel.

\begin{tabular}{rrrrrrr}
\hline Mol. Nos. & distance & Coulomb & polarization & dispersion & repulsion & interaction energy \\
3, 4 & 7.54 & -84.9 & -28.7 & -17.1 & 65.6 & -65.1 \\
2,3 & 7.492 & -105.5 & -68.6 & -20.3 & 144.9 & -49.4 \\
1,9 & 5.365 & -41.2 & -13.1 & -19.6 & 38.3 & -35.6 \\
1,8 & 4.945 & -7.8 & -4 & -18.2 & 13.6 & -16.4 \\
1,2 & 5.218 & -4.7 & -1.9 & -14.3 & 7.9 & -13.1 \\
5,7 & 5.151 & -0.9 & -1.3 & -14 & 5.6 & -10.7 \\
1,3 & 5.151 & -0.7 & -3 & -19.2 & 12.4 & -10.5 \\
& Totals & -107.8 & -55.4 & -87.4 & 142.8 & $-107.8^{*}$ \\
\hline
\end{tabular}

* This the calculated lattice energy

\subsection{Effects of temperature cycling and tailor-made additives}

Eight successive temperature cycles in which the dissolution steps reduce needle length more than the crystal growth steps increase it should ultimately give a more equant crystal product. This is observed as the crystal aspect ratio in temperature cycling runs without any additive shows a modest reduction in aspect ratio from an initial value of 4.8 to 3.2, Figure 5 .

The addition of low concentrations $(0.7 \mathrm{w} \%)$ BEN to the crystallizing solution subjected to temperature cycles allows control of BZA-INA product aspect ratio. After eight successive cycles, more equant crystals are observed, with a final aspect ratio of 1.51 . When the BEN concentration is increased to $10 \%$ thermal cycling has only a small effect on the aspect ratio because this high level of additive has reduced the aspect ratio from the start of thermal cycling. The results for no additive and NIC at $0.7 \%$ are very similar, Figure 3(c) and (d) and 
Figure S5 esi. In both cases the aspect ratio reductions are similar and crystal growth, as measured by crystal length, is suppressed to some extent by NIC. It is also interesting that the crystal aspect ratios have an observable effect on XRPD peak intensity due to preferred orientation. The intensity of the peak close to $17^{\circ}$ is enhanced in the BZA-INA samples which have needle crystals, Figure S4 esi. This peak is due to reflection from the $\left(\begin{array}{lll}-4 & 0 & 2\end{array}\right)$ plane, which is parallel to the needle axis, and thus will be enhanced in reflection mode by a needle morphology. The intensity of the -402 reflection is closer to the calculated intensity in the samples crystallized in the presence of BEN and NA in which needle growth was suppressed.

It was shown in section 3.3.1 that the additive BEN is incorporated into the crystal structure of the product. The additive would be expected to have maximum effect and be preferentially incorporated into the crystals at the potential needle tips where growth is fastest in the absence of an additive. There is also the potential problem that with an $0.7 \%$ initial concentration and continued additive incorporation its concentration could fall making it less effective. However, temperature cycling should have the ability to release incorporated additive in each dissolution step and help keep its solution concentration effectively constant making the additive effectively an aspect ratio catalyst rather than a reagent.

In the case of PABA, Raman and FT-IR spectroscopy showed that the additive crystallized concomitantly and was not incorporated into the BZA-INA lattice. The failure to incorporate PABA into the lattice is very likely due to the presence of one extra non- $\mathrm{H}$ atom in the molecule relative to INA and without its incorporation into the lattice no effect on morphology was observed. In the case of NIC it is likely that due to its structural similarity to BZA, direct substitution of BZA in the crystal is tolerated by the overall structure and thus there is no effect on crystal product morphology. The failure of PIC to effect morphology may be due to the lower energy calculated for its $R_{2}^{2}(8)$ adduct with INA. The calculated energy of the adduct is $10 \%$ lower than that of the INA dimer and thus it may be unable to compete with INA in the formation of $R_{2}^{2}(8)$ bridged hydrogen bonds, more detail in Table S1, esi. The observation that INIC blocks crystal growth is probably because it can replace INA very effectively in the INA dimer. The binding energy in the INIC-INA $R_{2}^{2}(8)$ adduct is $7 \%$ higher than of the INA dimer. While this adduct can be capped by BZA it cannot provide the hydrogen bond to another super molecule unit Figure 10(b), and this lack of bonding sites effectively blocks growth of the BZA-INA cocrystal lattice. 


\section{Conclusions}

In this article, we demonstrate how deep temperature cycling combined with additive addition can have a significant role in preventing the formation of a needle-like API product. This method can be used in the pharmaceutical industry to avoid the problems associated with needles which include filtration and general downstream processing. Using a carefully chosen tailor made additive a severe disruption of crystal shape can be induced if an important interaction in the crystal structure is blocked. It is also important to point out that the incorporation level of the additive in the product solid phase is very low and only a small percentage of its solution concentration in the process.

Cocrystals of benzoic acid and isonicotinamide grow as extreme needles from ethanol solution. The crystals have a slipped stack structure which is common to systems which show needle growth. Temperature cycling of a suspension of the crystals gives a modest reduction in crystal aspect ratio and an increase in crystal size. The experimental data shown in Figure 5 indicate that significant changes occur during the first four of the eight cycles that were carried out and the use of four cycles would seem efficient as after that point only minor changes are observed. Temperature cycling in the presence of the tailor-made additives benzamide or nicotinic acid at $0.7 \%$ weight concentration relative to BZA-INA greatly increases the effect on the aspect ratio, which is reduced to around 1. Raman and FTIR studies show that additives effective in suppressing needle growth were incorporated into the cocrystal structure and that they did not crystallize concomitantly. The multiplicative effect observed between thermal cycling and additive addition is probably due to repeated release of the incorporated additive during dissolution steps. This effectively restores additive concentration and makes the additive action catalytic. The combined use of thermal cycling and additive addition for crystal morphology control is likely to have general application to adjust product quality aspects such as crystal morphology.

\section{Acknowledgments}

This publication has emanated from research supported in part by a research grant from Science Foundation Ireland (SFI) and is co-founded under the European Regional Development Fund under Grant Number 12/RC/2275.

FC thanks the Crystallize COST Action CM1402 for travel funding of the Short-Term Scientific Mission (STSM). FC gratefully acknowledges the hospitality that he enjoyed as a 
Visiting researcher during his STSM at the EPSRC Centre for Innovative Manufacturing in Continuous Manufacturing and Crystallisation (CMAC) at the University of Strathclyde.

JtH, SJU and VS would like to thank EPSRC Centre for Innovative Manufacturing in Continuous Manufacturing and Crystallisation (Grant Ref EP/K503289/1) for funding this work. The authors would like to acknowledge that part of this work was carried out in the CMAC National Facility supported by UKRPIF (UK Research Partnership Fund) award from the Higher Education Funding Council for England (HEFCE) (Grant Ref HH13054).

\section{References}

(1) Adobes-Vidal, M.; Maddar, F. M.; Momotenko, D.; Hughes, L. P.; Wren, S. A.; Poloni, L. N.; Ward, M. D.; Unwin, P. R., Face-discriminating dissolution kinetics of furosemide single crystals: in situ three-dimensional multi-microscopy and modeling. Cryst. Growth Des. 2016, 16, 4421-4429.

(2) Tung, H.-H., Industrial Perspectives of Pharmaceutical Crystallization. Org. Process Res. Dev. 2013, 17, 445-454.

(3) Muller, P., Glossary of terms used in physical organic chemistry (IUPAC Recommendations 1994). In Pure Appl. Chem., ed.; 1994; Vol. 66, p 1077.

(4) Chen, J.; Trout, B. L., Computer-aided solvent selection for improving the morphology of needle-like crystals: A case study of 2, 6-dihydroxybenzoic acid. Cryst. Growth Des. 2010, 10, 4379-4388.

(5) Chen, J.; Sarma, B.; Evans, J. M. B.; Myerson, A. S., Pharmaceutical Crystallization. Cryst. Growth Des. 2011, 11, 887-895.

(6) Brittain, H. G., Pharmaceutical Cocrystals: The Coming Wave of New Drug Substances. J. Pharm. Sci. 2013, 102, 311-317.

(7) Pallipurath, A. R.; Civati, F.; Eziashi, M.; Omar, E.; McArdle, P.; Erxleben, A., Tailoring Cocrystal and Salt Formation and Controlling the Crystal Habit of Diflunisal. Cryst. Growth Des. 2016, 16, 6468-6478.

(8) Jiang, M.; Zhu, X.; Molaro, M. C.; Rasche, M. L.; Zhang, H.; Chadwick, K.; Raimondo, D. M.; Kim, K.-K. K.; Zhou, L.; Zhu, Z.; Wong, M. H.; O'Grady, D.; Hebrault, D.; Tedesco, J.; Braatz, R. D., Modification of Crystal Shape through Deep Temperature Cycling. Ind. Eng. Chem. Res. 2014, 53, 5325-5336.

(9) Wu, Z.; Yang, S.; Wu, W., Application of temperature cycling for crystal quality control during crystallization. CrystEngComm 2016, 18, 2222-2238.

(10) Carless, J. E.; Foster, A. A., Accelerated crystal growth of sulphathiazole by temperature cycling. J. Pharm. Pharmacol. 1966, 18, 697-708.

(11) van Westen, T.; Groot, R. D., Effect of Temperature Cycling on Ostwald Ripening. Cryst. Growth Des. 2018, 18, 4952-4962.

(12) Suwannasang, K.; Flood, A. E.; Rougeot, C.; Coquerel, G., Using Programmed Heating-Cooling Cycles with Racemization in Solution for Complete Symmetry Breaking of a Conglomerate Forming System. Cryst. Growth Des. 2013, 13, 3498-3504. 
(13) Li, W. W.; Spix, L.; de Reus, S. C. A.; Meekes, H.; Kramer, H. J. M.; Vlieg, E.; ter Horst, J. H., Deracemization of a Racemic Compound via Its Conglomerate-Forming Salt Using Temperature Cycling. Cryst. Growth Des. 2016, 16, 5563-5570.

(14) Simone, E.; Steele, G.; Nagy, Z. K., Tailoring crystal shape and polymorphism using combinations of solvents and a structurally related additive. CrystEngComm 2015, 17, 93709379.

(15) Heffernan, C.; Ukrainczyk, M.; Zeglinski, J.; Hodnett, B. K.; Rasmuson, Å. C., Influence of Structurally Related Impurities on the Crystal Nucleation of Curcumin. Cryst. Growth Des. 2018, 18, 4715-4723.

(16) Weissbuch, I.; Leiserowitz, L.; Lahav, M., "Tailor-Made Additives", and Impurities. In Crystallization Technology Handbook, Mersmann, A., Ed. CRC Press: Boca Raton 2001; p 54.

(17) Lee, S.-H.; Lee, G.-H.; Lee, K.-H.; Jazbinsek, M.; Kang, B. J.; Rotermund, F.; Kwon, O. P., In Situ Tailor-Made Additives for Molecular Crystals: A Simple Route to Morphological Crystal Engineering. Cryst. Growth Des. 2016, 16, 3555-3561.

(18) Cai, Z.; Liu, Y.; Song, Y.; Guan, G.; Jiang, Y., The effect of tailor-made additives on crystal growth of methyl paraben: Experiments and modelling. J. Cryst. Growth 2017, 461, 19.

(19) Keshavarz, L.; Steendam, R. R. E.; Blijlevens, M. A. R.; Pishnamazi, M.; Frawley, P. J., Influence of Impurities on the Solubility, Nucleation, Crystallization, and Compressibility of Paracetamol. Cryst. Growth Des. 2019, 19, 4193-4201.

(20) Simone, E.; Klapwijk, A. R.; Wilson, C. C.; Nagy, Z. K., Investigation of the Evolution of Crystal Size and Shape during Temperature Cycling and in the Presence of a Polymeric Additive Using Combined Process Analytical Technologies. Cryst. Growth Des. 2017, 17, 1695-1706.

(21) Hatcher, L. E.; Li, W.; Payne, P.; Benyahia, B.; Rielly, C. D.; Wilson, C. C., Tuning Morphology in Active Pharmaceutical Ingredients: Controlling the Crystal Habit of Lovastatin through Solvent Choice and Non-Size-Matched Polymer Additives. Cryst. Growth Des. 2020, 20, 5854-5862.

(22) Svoboda, V.; MacFhionnghaile, P.; McGinty, J.; Connor, L. E.; Oswald, I. D. H.; Sefcik, J., Continuous Cocrystallization of Benzoic Acid and Isonicotinamide by MixingInduced Supersaturation: Exploring Opportunities between Reactive and Antisolvent Crystallization Concepts. Cryst. Growth Des. 2017, 17, 1902-1909.

(23) Seaton, C. C.; Parkin, A.; Wilson, C. C.; Blagden, N., Growth of an Organic CoCrystal upon a Component Subphase. Cryst. Growth Des. 2008, 8, 363-368.

(24) Svoboda, V. Continuous crystallization of multicomponent materials. University of Strathclyde, $\mathrm{PhD}$ thesis, 2018.

(25) Aakeröy, C. B.; Beatty, A. M.; Helfrich, B. A., "Total Synthesis" Supramolecular Style: Design and Hydrogen-Bond-Directed Assembly of Ternary Supermolecules. Angew.Chem. Int. Ed. 2001, 40, 3240-3242.

(26) Park, G. Y.; Lee, J. Y.; Himes, R. A.; Thomas, G. S.; Blackburn, N. J.; Karlin, K. D., Copper-Peptide Complex Structure and Reactivity When Found in Conserved His-Xaa-His Sequences. J. Am. Chem. Soc. 2014, 136, 12532-12535.

(27) Elts, E.; Greiner, M.; Briesen, H., In Silico Prediction of Growth and Dissolution Rates for Organic Molecular Crystals: A Multiscale Approach. Crystals 2017, 7, 288. 
(28) O'Mahony, M.; Seaton, C. C.; Croker, D. M.; Veesler, S.; Rasmuson, A. C.; Hodnett, B. K., Investigating the dissolution of the metastable triclinic polymorph of carbamazepine using in situ microscopy. CrystEngComm 2014, 16, 4133-4141.

(29) Leyssens, T.; Baudry, C.; Escudero Hernandez, M. L., Optimization of a Crystallization by Online FBRM Analysis of Needle-Shaped Crystals. Org. Process Res. Dev. 2011, 15, 413-426.

(30) Gavezzotti, A., Efficient computer modeling of organic materials. The atom-atom, Coulomb-London-Pauli (AA-CLP) model for intermolecular electrostatic-polarization, dispersion and repulsion energies. New J. Chem. 2011, 35, 1360-1368.

(31) McArdle, P., Oscail, a program package for small-molecule single-crystal crystallography with crystal morphology prediction and molecular modelling. J. Appl. Crystallogr. 2017, 50, 320 - 326.

(32) Seaton, C. C.; Parkin, A.; Wilson, C. C.; Blagden, N., Controlling the Formation of Benzoic Acid: Isonicotinamide Molecular Complexes. Cryst. Growth Des. 2009, 9, 47-56.

(33) Al-Zoubi, N.; Koundourellis, J.; Malamataris, S., FT-IR and Raman spectroscopic methods for identification and quantitation of orthorhombic and monoclinic paracetamol in powder mixes. J. Pharm. Biomed. Anal. 2002, 29, 459-467.

(34) Agatonovic-Kustrin, S.; Rades, T.; Wu, V.; Saville, D.; Tucker, I. G., Determination of polymorphic forms of ranitidine-HCl by DRIFTS and XRPD. J. Pharm. Biomed. Anal. 2001, 25, 741-750.

(35) Donnay, J. D.; Harker, D., A new law of crystal morphology extending the law of Bravais. Amer. Mineral. 1937, 22, 446-447.

(36) Panina, N.; van de Ven, R.; Janssen, F. F. B. J.; Meekes, H.; Vlieg, E.; Deroover, G., Study of the Needle-Like Morphologies of Two $\beta$-Phthalocyanines. Cryst. Growth Des. 2008, 9, 840-847.

(37) Piana, S.; Reyhani, M.; Gale, J. D., Simulating micrometre-scale crystal growth from solution. Nature 2005, 438, 70-73.

(38) Panina, N.; Van de Ven, R.; Janssen, F.; Meekes, H.; Vlieg, E.; Deroover, G., Study of the needle-like morphologies of two $\beta$-phthalocyanines. Cryst. Growth Des. 2009, 9, 840847.

(39) Dove, P. M.; Han, N. In Kinetics of mineral dissolution and growth as reciprocal microscopic surface processes across chemical driving force, AIP Conf. Proc., 2007; American Institute of Physics: 2007; pp 215-234.

(40) Clark, J. N.; Ihli, J.; Schenk, A. S.; Kim, Y.-Y.; Kulak, A. N.; Campbell, J. M.; Nisbet, G.; Meldrum, F. C.; Robinson, I. K., Three-dimensional imaging of dislocation propagation during crystal growth and dissolution. Nat. Mater. 2015, 14, 780-784.

(41) Civati, F.; Erxleben, A.; Kellehan, S.; McArdle, P., Conversion of Gel-Forming Crystal Needles To Easily Processable More Equant Crystals Using High-Shear Ultralow Attrition Agitation: Accelerated Ostwald Ripening without Crystal Attrition. Cryst. Growth Des. 2019, 19, 1502-1504.

(42) Walshe, N.; Crushell, M.; Karpinska, J.; Erxleben, A.; McArdle, P., Anisotropic Crystal Growth in Flat and Nonflat Systems: The Important Influence of van der Waals Contact Molecular Stacking on Crystal Growth and Dissolution. Cryst. Growth Des. 2015, $15,3235-3248$.

(43) Gavezzotti, A., Calculation of lattice energies of organic crystals: the PIXEL integration method in comparison with more traditional methods. Z. Kristallogr. Cryst. Mater. 2005, 220, 499-510. 


\section{For Table of Contents Use Only}

Francesco Civati ${ }^{\mathrm{a}}$, Vaclav Svoboda ${ }^{\mathrm{b}}$, Stephanie J. Urwin ${ }^{\mathrm{c}}$, Patrick McArdle ${ }^{\mathrm{a}}$, Andrea Erxleben $^{\mathrm{a},{ }^{*}}$, Denise Croker ${ }^{\mathrm{d}}$, Joop H. ter Horst ${ }^{\mathrm{c}}{ }^{,}$

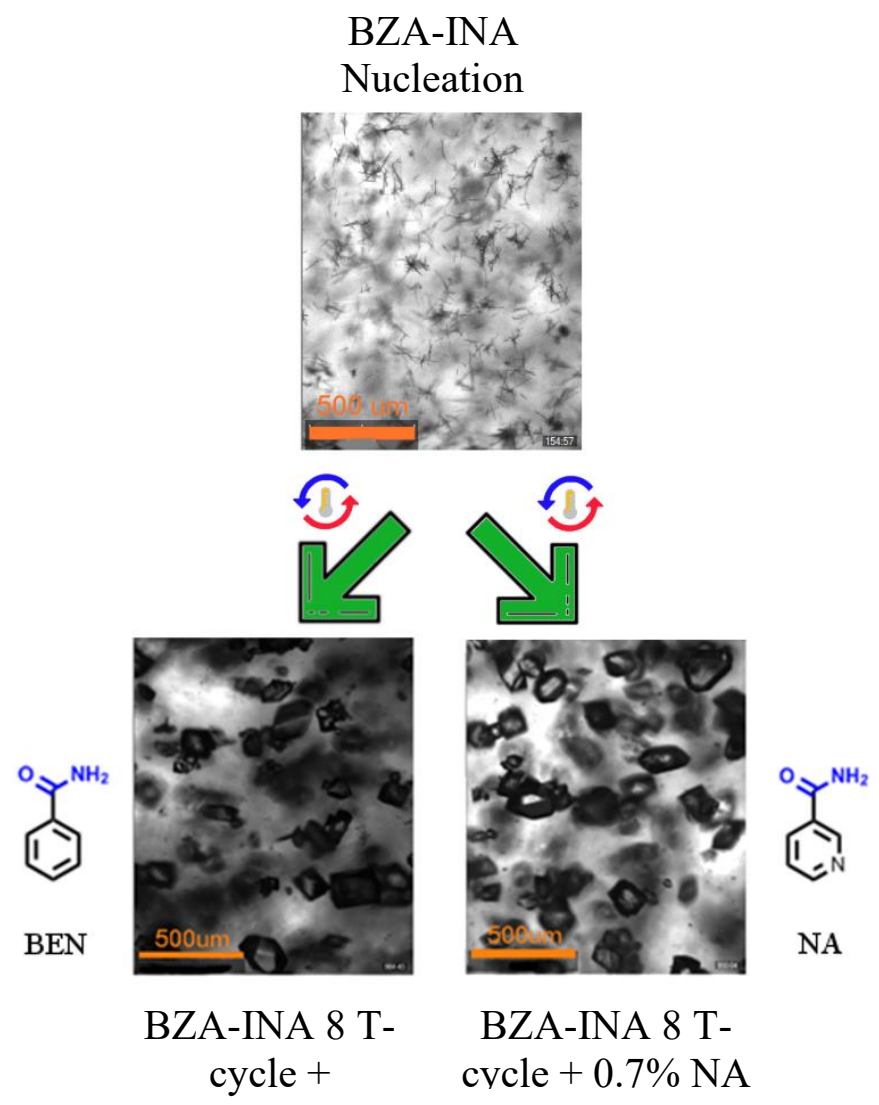

\section{SYNOPSIS}

The simultaneous use of temperature cycling and tailor-made additives offers a new and effective approach for morphology control of a pharmaceutical cocrystal. 
